\title{
Delivering communication strategy training for people with aphasia: what is current clinical practice?
}

Firle Beckley, Wendy Best, Suzanne Beeke

Division of Psychology and Language Sciences, University College London

Chandler House, 2 Wakefield Street, London WC1N 1PF

Address correspondence to: Firle Beckley, phone number 0207679 4255; e-mail: f.beckley@ucl.ac.uk

Keywords: Communication strategies, aphasia, clinical practice, intervention, conversation.

\begin{abstract}
Background: Communication Strategy Training (CST) is a recognized part of UK speech and language therapists' (SLT) role when working with a person with aphasia (PwA). Multiple CST interventions have been published but, to date, there are no published studies exploring clinical practice in this area.

Aim: To investigate UK SLTs' current CST practices.

Methods \& Procedures: 37 UK SLTs completed an online questionnaire, eight of which attended a follow-up focus group. A clinical consistency scale was applied to the questionnaire data and tasks that were most consistently used were explored in
\end{abstract}


the focus group and analyzed using a primarily deductive thematic data analysis approach.

Outcomes \& Results: Three key CST findings arose: (i) the rarity with which SLTs focus equally and explicitly on both communication partners' strategies (iii) SLTs' differing understandings of CST terminologies and concepts and underuse of formal assessment (iii) the absence of video feedback.

Conclusion \& Implications: This study's survey findings suggest that CPs not only receive half the amount of CST given to PwAs, but they also play a more passive learning role when they are present. This is an interesting point to consider when the current evidence base contains stronger evidence for the effectiveness of $C P$-CST over other CST approaches, describing it as "an effective method" that "may be maintained over time" (Simmons-Mackie, Raymer, Armstrong, Holland, \& Cherney, 2010, p. 1833).

What this paper adds?

Despite the variety of published CST approaches available to SLTs, current clinical practice in this area has not been explored. The value of this study is that is opens up a dialogue between researchers and clinicians; the next important step is to consider what we do with these lessons.

\section{Introduction}

Living successfully with aphasia has been linked to a person's opportunities to participate in meaningful activities and maintain close relationships despite linguistic impairments (Brown, Worrall, Davidson, \& Howe, 2012). Communication strategies, or compensatory strategies, are one way in which a person with aphasia (PWA) and their conversation partner(s) (CPs) can circumvent the communication barriers imposed by aphasia.

A communication strategy (also known as a compensatory strategy) is defined as: "A new or expanded communicative behaviour, often spontaneously acquired and 
systematically employed, to overcome a communication barrier in an effort to meet both transactional and interactional communicative goals" (Simmons-Mackie \& Damico, 1997 p.770).

As Simmons-Mackie and Damico (1997) highlight communication functions not just to transact information but also to facilitate social interaction. A PwA and CP may use verbal and non-verbal communication strategies to achieve these functions. Typical strategies a PwA may use include circumlocution, keywords signing, gesture, pantomime, drawing, writing, communication charts, and electronic aids (Beeke et al., 2015; Simmons-Mackie \& Damico, 1997). Typical CP strategies can include conversation continuers such as 'mm hum' (Wilkinson, Lock, Bryan, \& Sage, 2011) and writing, gesture, drawing and resource materials (Kagan, 1998).

In the UK, assessment and treatment of communication strategy use is a recognized part of the role of a speech and language therapist (SLT) (Royal College of Physicians Intercollegiate Stroke Working Party, 2012). There is a diverse literature of therapies targeting communication strategy use, henceforth referred to collectively as Communication Strategy Training (CST). All encourage the use of communication strategies to compensate for aphasia and can be broadly grouped into three main approaches: (i) Functional CST (F-CST) targeting a PwA's strategy use in everyday transactional communicative situations (Armstrong, Ferguson, \& Simmons-Mackie, 2013); (ii) Communication Partner CST (CP-CST), targeting CPs' strategy use as a means to reducing the psychosocial consequences of aphasia (Kagan, 1998; Simmons-Mackie, 2008) and (iii) Conversation (also known as Interactional) CST (C-CST) targeting a $\mathrm{CP}$ or $\mathrm{CP}$ and PwA's strategy use within their everyday conversations (Wilkinson, 2010). A review of these approaches and a discussion about current clinical CST practices will now follow.

F-CST focuses on the transaction of information and aims for a PwA to generalize multi-modal strategies taught using highly structured tasks into the real world. Interventions typically comprise the SLT and PwA practising strategies using highly structured picture and object description and role-play tasks either one to one (for example Promoting Aphasics' Communicative Effectiveness- (Davis \& Wilcox, 1985) or in a group (Rautakoski, 2011). However, the research directly exploring 
generalization found it variable and context-dependent. Purdy, Duffy, \& Coelho's, (1994) 15 subjects persisting with ineffective verbal output in structured conversation tasks despite successful non-verbal strategy use in therapy and Yoshihata, Watamori, Chujo, \& Masuyama, (1998) only detecting generalization in the presence of a skilled CP who facilitated the PwAs' trained strategy use.

The importance of the $\mathrm{CP}$ in revealing a PwA's skills comes to the forefront in $\mathrm{CP}$ CST. Defined as "an intervention directed at people other than the person with aphasia with the intent of improving the language, communication, participation, and/or well being of the person with aphasia" (Simmons-Mackie et al., 2010, p. 1814). CP-CST embraces the psychosocial model of aphasia, championing the influence of the CP and wider environment on a PwA's ability to 'live well' with aphasia (Simmons-Mackie, 2008). 'Living well' is defined as the personal adjustments and life-style changes needed to enhance life participation (SimmonsMackie, 2008; Simmons-Mackie, Raymer, Armstrong, Holland, \& Cherney, 2010). Key to CP-CST is a belief that communicative success is not the sole responsibility of the PwA; rather it is the role of the $\mathrm{CP}$ to reveal a PwA's communicative competence (e.g. Supported Conversation for Adults with Aphasia SCA, Kagan, 1998).

In 2010 a systematic review concluded CP-CST to be an "effective method of improving partner skill” (Simmons-Mackie et al., 2010, p. 1833). However, the same review also concluded that CP-CST is only "probably effective" at enhancing a PwA's activity and participation (Simmons-Mackie et al., 2010, p. 1833). As Simmons-Mackie et al (2010) discuss, this may reflect a lack of rigorous study of PwA outcomes. Alternatively it could reflect the unintentional dependence of a PwA on a skilled CP that CP-CST may cause. Conversation is, by definition, an interaction between two or more people (Simmons-Mackie, Savage, \& Worrall, 2014) where who speaks, when they speak and the size of their turn is not predetermined (Wilkinson, 2010). In contrast, institutional interactions (i.e. those between a trained professional and client) are led by the professional using questions and controlling the topic (Wilkinson, 2010). It could be argued that training the CP in the absence of training the PWA might risk the PWA's participation in conversation, 
making them solely dependent on an individual CP, which could longer term impact their self-efficacy i.e. their belief or confidence in their own abilities (Bandura, 1994)

The third approach, C-CST, specifically embraces the unstructured two-way nature of everyday conversation and increasingly treats the CP and PwA equally. It is an approach being actively researched in the UK, the US and Scandinavia (see Simmons-Mackie et al., 2014 for most recent review) and it aims to explicitly change communication strategy use by working directly within everyday conversations (Simmons-Mackie et al., 2014). Therapy often includes playing clients video-clips of their conversations to raise insight into current behaviour and facilitate change (Beckley et al., 2013; Lock, Wilkinson, \& Bryan, 2001). Promising changes in the communication strategy use of both a PwA and their CP within everyday conversation have been reported post C-CST focused on both partners (Beeke et al., 2015; Wilkinson et al., 2011), suggesting that C-CST's context driven, collaborative approach could be an effective means of maximizing a PwA's communication strategy learning, and generalization into everyday conversation.

To the best of the author's knowledge there are no published papers directly exploring UK SLTs' CST practices. However a Swedish study (Johansson, Carlsson, \& Sonnander, 2011) and a smaller Canadian study (Hallé, Le Dorze, \& Mingant, 2014) touched on CST practices when they explored SLT practices with family members. Johansson et al's (2011) survey of 206 SLTs revealed 48\% of respondents met with families, on average 2-3 times, with only $17 \%$ of these encounters dedicated to CST for families. Similarly low levels of CP-CST were noted in Hallé et al's. (2014) study; just one of eight participants in their semi-structured interview described frequently observing the "conversations the person with aphasia had with a family member for 'excesses' and 'absences' in the dyad's communication" (Hallé et al., 2014, p. 757). The other SLTs primarily focused on the PwA's linguistic impairment and communicative success. Both studies suggested that health service time pressures, and SLTs' concerns about when and how best to train CPs, played a role in the absence of CPs from CST.

Neither of these studies specifically asked which CST approaches SLTs used nor how they carried them out in practice. As a result it is not possible to ascertain the 
extent to which the current evidence base for CST influenced SLTs who took part in these studies. As to why SLTs expressed concerns about training CPs, it is possible that, in addition to familiarity with the evidence base, SLT education practices across these countries may be relevant, as might structures in place for the delivery of healthcare. Furthermore it is unclear whether these findings are applicable to UK practice. Thus the work reported here directly investigates UK SLTs' understanding of what is meant by CST and their current CST practices. SLTs were asked to participate in a questionnaire and a follow-up focus group. The questionnaire aimed to answer three research questions: (1) How are SLTs assessing communication strategy skills? (2) How are communication strategies being worked on in therapy? (3) Is there a typical structure to CST sessions? The focus group aimed to explore the techniques SLTs use within the most consistently reported intervention tasks, and the reported barriers to video use in CST. This paper first presents a quantitative analysis of the questionnaire data, focusing on the most consistently reported assessment and intervention tasks, and the prevalence of video use during CST. This is followed by a qualitative analysis of focus group data.

\section{Methods}

This work is part of an ongoing project funded by the Stroke Association (Project Reference JTRF 2012/01) to compare the effectiveness of CST as routinely provided by SLTs with a published C-CST intervention called Better Conversations with Aphasia (BCA) (Beeke et al., 2013). The work presented here aims to provide an insight into current practice in order to inform later work, which will develop a CST programme reflecting current UK SLT practices in the field of aphasia rehabilitation. Findings from stages 1 and 2 of the CST development process will be reported here (figure 1).

A description of stages 1 and 2 of the design process are provided first, followed by details of the recruitment process and finally the data analysis methodology.

Figure 1 here 
The first author devised the questionnaire, focusing questions on issues derived from the evidence base for CST, and drawing on clinical experience. Questions covered: (1) Professional background; (2) Communication strategy assessment practices; and (3) Communication strategy intervention practices. The questionnaire requested SLTs report assessments and interventions used in the past 12 months. It was piloted by three experienced SLTs, resulting in the separate sections for each CST approach being collapsed into two universal assessment and intervention sections, halving the number of questions and reducing repetition. The final version contained 37 closed questions presenting lists of formal and informal assessment and therapy options that required multiple choice or rating-scale responses. Four questions were open for free-text responses. Definitions of key terminology were given to ensure a shared understanding of the concepts to be explored (see table 1). Listed options came from published sources e.g. SCA (Kagan, 1998), PACE (Davis \& Wilcox, 1985) and also drew on the clinical experience of the first author. Rating scales took the following format: 'always do this/ do this with most people/ occasionally do this/ rarely do this/ never do this', or 'all of the time/ about $75 \%$ of the time/ about $50 \%$ of the time/ about $25 \%$ of the time/ less than $25 \%$ of the time/ never'. For a summary of the questions analyzed in this paper to elicit answers to the three main research questions, and the recruitment procedure, see Appendix A.

Table 1 here

\section{Stage 2: Focus Group}

A 2-hour focus group, designed and moderated by the first author, was held at University College London (UCL) with an assistant moderator, a UCL student SLT. It explored in greater depth the second research question: how are communication strategies being worked on in therapy? To ensure the validity of the group discussion (i.e. that clinicians discussed their actual rather than ideal practice), the moderator withheld evidence based best practice information (see Murphy et al., 1998 for 
details). Discussion centred on education tasks (question 34) and communication strategy practice tasks (question 37) that the questionnaire respondents stated they used with a moderate or high degree of consistency (see below for further details). This information, provided in graph form, alongside group member's examples of communication strategy sheets used with clients, acted as a catalyst for discussion about specific therapy techniques. Finally the group was asked why reported video use in CST was so low.

The focus group was videotaped, and the student SLT took field notes. The first author then created an abridged transcript, omitting talk not directly related to therapy practices.

\section{Recruitment of questionnaire participants}

Only UK SLTs currently practicing clinically in aphasia rehabilitation were recruited. Clinically inactive SLTs and student SLTs were excluded, as it was felt their views would not reflect current practice.

An invitation to complete the questionnaire was emailed to: 484 SLTs registered with either the UCL Aphasia Research Group (ARG); the UCL Better Conversations with Aphasia (BCA) project database; or were personal contacts of the first author. A questionnaire link was also posted on the first author's blog (www.firleb.wordpress.com) and the ARG blog (www.aphasiaresearch.wordpress.com). The questionnaire was open for responses from the $10^{\text {th }}$ June to the $15^{\text {th }}$ July 2013 in line with the first authors PhD study timeline. All respondents who completed the questionnaire were then invited to participate in follow-up focus groups, 12 SLTs volunteered to take part, of eight whom were able to attend UCL on the same day.

According to UCL Research Ethics guidelines this study was a service evaluation using anonymised data that would not cause substantial damage and distress. Ethical approval was not required. Nevertheless consent was gained from focus group participants to store the videoed group data until 1 year after the first author's $\mathrm{PhD}$ completion. 


\section{Data Analysis}

Below is a description of the two main data analysis methodologies used: (i) categorical data analysis of closed questions with the questionnaire, and (ii) qualitative analysis of the questionnaire's open questions and focus group data.

\section{(i) Categorical data analysis}

The questionnaire asked SLTs about assessment and therapy practices, distinguishing between functional and conversational contexts. However, responses across these contexts were similar, so were analysed together.

The most commonly used CST practices were determined by calculating the percentage of SLTs selecting each rating or multiple-choice option for the closed questions (see Appendix B). Where there was a large spread across ratings, categories were collapsed into larger groups (see Mathers-Schmidt \& Kurlinski 2003 for a similar approach). For example the wide variety of responses to question 37 regarding the frequency of use of practice tasks lead to the 6-point rating scale being collapsed into a 2-point scale 'used $50 \%$ of the time or more' and 'used $25 \%$ of the time or less' in order to gain an overall impression of practice.

Mathers-Schmidt \& Kurlinski's (2003) clinical consistency scale was used to determine the consistency with which questionnaire respondents reported using a clinical practice at the same level of frequency. Thus a frequency level selected by: $75 \%$ of respondents is a highly consistent practice; $50-75 \%$ of respondents is a moderately consistent practice, and less than $50 \%$ of respondents is an inconsistent practice.

\section{(ii) Qualitative data analysis}

For the purpose of this study question 35 of the questionnaire, requesting two examples of information provision to a PwA and CP, (see Appendix A) and the focus group data (see Appendix B) were subject to a primarily deductive thematic data analysis (with coding categories taken from the intervention literature, see below), 
supplemented by an inductive approach to account for data that remained uncoded (Fereday \& Muir-Cochrane, 2006). The abridged focus group transcript, and information examples were uploaded into QRS NVivo 10, a computer assisted qualitative data analysis software (CAQDAS) for analysis. Analysis comprised of the following steps, and was carried out by the first author. Where applicable examples are illustrated with quotes detailing: the time they were said; or question which generated it; and who said it, labeled anonymously- P1 to P8 for the focus group or with a six-digit figure for question 35 .

Step 1: Pre-determined task headings based on common clinical terminology in the field of aphasia and published definitions where available (see Appendix C) were entered as separate nodes in NVivo and the first author coded uploaded text by categorizing descriptions under relevant nodes. For example "It's a barrier between you and me. You have some information on your side and I have some information on my side and you need to communicate to me" (00:32:16 P2) was categorized under the node "barrier task".

Step 2: Task descriptions that were not covered by this deductive coding system were placed under a node labeled "additional tasks". These descriptions were reviewed and placed under two inductive codes by the first author called 'real-life tasks' and 'structured communication tasks' (see Appendix C).

Step 3: Each node was re-examined for examples of techniques used to complete each task. Examples were coded according to deductive labels based on published definitions (e.g. modeling, feedback and prompting). Relevant examples that did not match published definitions were, where appropriate, given inductive codes (e.g. linking new knowledge to client's real-life experiences was defined as 'bridging', see Appendix D). Where several techniques were referenced, descriptions were coded under multiple technique nodes. Thus, this description of a real-life task was coded as an example of two therapy techniques: collaboration and environmental restructuring: 
"It's the carer or partner who'll go in and clue in a shopkeeper and 'so he's going to come in with a 220 note and er, he doesn't talk but he understands just fine, you know, can you help him out?'”' (00:29:33 P2).

The first author's coded techniques were reliability checked, by the last author, unblinded, against the list of technique definitions. Where both authors disagreed on the first author's coding, descriptions were removed before step 4 of the analysis.

Step 4: An NVivo 10 matrix-coding query was used to determine the frequency with which each technique was referenced within a task category. The number of technique counts were entered into excel from which frequency bar charts were generated (Figure 2).

Figure 2 here

\section{Results}

Characteristics of participants

Thirty-seven SLTs completed the questionnaire, eight of whom went on to participate in the focus group. Table 2 details participant profiles at each stage of the methodology. Over half of questionnaire respondents (62\%) were SLTs working in a community or mixed community/acute setting; $89 \%$ had worked clinically for more than 1 year, and $54 \%$ for more than 5 years. People with aphasia accounted for 25 $75 \%$ of the caseload for $65 \%$ of questionnaire respondents.

Over half of focus group members $(62 \%)$ had worked in acute stroke services for more than 1 year, with 50\% having over 10 years clinical experience. Of those that responded, $67 \%$ said that clients with aphasia accounted for $50-75 \%$ of their caseload.

Table 2 here

Questionnaire Findings 
This section first describes the typical structure of CST followed by an examination of which intervention tasks are most consistently used. It finally reports on the frequency of video use within CST.

\section{(i) Typical structure of CST interventions}

The questionnaire revealed greater consistency between SLTs regarding the overall structure of CST when targeting a CP in comparison to a PwA (Figure 3). SLTs demonstrated a moderate consistency in the number of assessment and therapy sessions offered to a CP with $70 \%$ offering $1-2$ assessment sessions and $55 \%$ offering 1-2 therapy sessions. This consistency was not seen for a PwA, with the most frequently selected offering of 1-2 assessment sessions remaining an inconsistent practice at $49 \%$. Further, the wide spread of responses in relation to the number of therapy sessions a PwA is offered, suggests they receive anything from 1 to over 4 sessions.

Figure 3 here

A similar pattern was seen regarding who delivers CST (Figure 4). SLTs deliver CST to a $\mathrm{CP}$ with moderate consistency $(70 \%)$, but delivery of CST to a PwA was inconsistently split between the SLT (49\%) and SLT plus an assistant (41\%).

Figure 4 here

In terms of therapy settings, the only consistent practice was the absence of CST delivery in outpatient settings (54\% of PwA and 62\% of $\mathrm{CP}$ receiving $\mathrm{CST}$ in alternative settings). The only setting that approached moderate consistency was delivery in the PwA's home.

Assessment practices revealed a high level of consistency regarding the use of informal assessment, with $84 \%$ of SLTs using PwA and/or CP self-report to assess communication strategy skills, and $76 \%$ using observation in a functional or conversational setting. SLTs also showed high consistency in their lack of use of the majority of formal assessment tools and methods, 78-97\% of SLTs never using 
published assessment tools such as the MPC/MSC (Kagan 2004) or SPPARC (Lock et al., 2001).

In summary, SLTs' only highly consistent practices were the use of informal assessment of communication strategy use and the non-use of formal assessments. In terms of the number of sessions they allocate to CST SLTs appear more consistent in their practices with CPs than PwA. There was moderate consistency in terms of the number of sessions and providers of CST for CPs. In contrast practices with a PwA were inconsistent, although 1 or 2 assessment sessions and undertaking CST in a PwA's home almost reached moderate consistency.

(ii) Intervention tasks used to deliver CST

SLTs were asked about the content and format of education tasks (e.g. written and or verbal information-giving about communication strategies, aphasia, communication, conversation). The questionnaire revealed that most educational materials are given in written and verbal formats. Figure 5 shows that provision of a tailored communication strategy list is a highly consistent practice, undertaken by $81 \%$ of SLTs, and that the provision of generic or tailored information about the impact of aphasia on communication and conversations is a moderately consistent practice, undertaken by $54 \%$ and $53 \%$ of SLTs respectively.

\section{Figure 5 here}

When asked about tasks for practising communication strategy there was so little consistency with regards to the frequency of their use that the 6-point scale was collapsed into two groups: (i) tasks used more than $50 \%$ of the time and (ii) tasks used $25 \%$ of the time or less. Figures 6 and 7 illustrate the top five tasks in each group. Of the five practice tasks used more than $50 \%$ of the time, only 'conversation with the SLT' (84\%) reached a high level of consistency. The tasks 'conversation with dyad' and 'picture description' reached moderate consistency used by $57 \%$ of the SLTs. The remaining tasks 'conversation coaching with the SLT' and 'barrier games' were inconsistently used by $43 \%$ and $38 \%$ of SLTs respectively. 
In contrast, SLTs were highly consistent in the five tasks used $25 \%$ of the time or less (Figure 7), strongly suggesting that these five are not a common feature of CST.

Figure 6 here

Figure 7 here

Finally SLTs were asked how often they use video as part of their therapy. $70 \%$ of SLTs never use video with a PwA and $81 \%$ never use it with a CP.

In summary, the questionnaire revealed a greater consistency among SLTs with regards to the type of information they supply to a PwA and/or CP. There was less consistency in the tasks deployed to practice strategy use. A tailored list of communication strategies is the most consistently provided piece of information, and practice is most consistently carried out during conversation with an SLT. Practice tasks used least are: role-play, PACE activities and barrier tasks. The wide variation in the frequency of used practice therapy tasks makes firm conclusions about 'typical' CST difficult to draw. However, video use is clearly not a feature.

Findings from the Focus Group and Q35

This section focuses on the techniques SLTs report using when undertaking the most consistently used therapy tasks, ascertained from the questionnaire. This section ends with SLTs' reported reasons for low video use within CST

(i) Education tasks and key related techniques

Information provided during education tasks includes: "advertising pretty widely that communication is more than just talk" (00:35:33 P2); "talking about the impact of 
the impairment...on relationships, on functional living in the house" (00:23:59 P7) and "how to communicate best with your partner" (00:20:03 P5).

As figure 8 shows, nine education techniques are mentioned, with the top three being: collaboration (28/88 references), modeling (13/88 references) and bridging (12/88 references).

Figure 8 here

Collaboration takes three main forms; the SLT (i) 'talking', or 'reading through' information with clients, (ii) modeling desired behaviours in front of the CP (iii) cocreating tailored information/strategy sheets with clients. The final seven references to collaboration were individual examples of different collaborative techniques, ranging from leaving written information for a $\mathrm{CP}$ and PwA to read together (SLT 736667-Q35) to the SLT carrying out a session and then getting "the CP to like assess it"' (00:54:39 P5).

Modeling was referred to by all focus group members and five questionnaire respondents made reference to this second most referenced education technique. At one extreme, P6 commented, “I don't give out information anymore" (00:29:51 P6), preferring to "have a conversation, a 'chat'...they're talking so I am drawing, writing down key words and referring back and putting a pen and paper there, not even saying" (00:30:44 P6). Nine of the thirteen references explicitly mentioned modeling to help the CP "see what works" (00:34:30 P3), these were also coded as collaborative.

Bridging, referred to twelve times, is the third most referenced education technique. The majority (seven) of those references describe SLTs "recalling” (SLT 740251 Q35) or "reinforcing" (00:45:46 P4) concrete examples of when a PwA or their CP "experienced" (SLT 730999-Q35) or successfully used communication strategies. P2, P4 and P5, also use analogies to everyday life to explain the abstract concept of aphasia. For example P4 refers to it as "like being in a foreign country...you don't understand what people say but you know what the situation is about" (00:20:58 P4). 
In summary, analysis of the top three education tasks reveals the complexity and diversity of these therapeutic interactions. The most frequently cited technique, collaboration, takes many forms and significantly overlaps with modeling, the second most cited education technique. Similarly bridging is also multifaceted, including reinforcing examples of the successful use of communication strategies in real-life and using everyday analogies to explain abstract or complex concepts, overlapping collaboration and modeling.

(ii) Tasks deployed for practicing communication strategy use

The focus group initially explored conversation and picture description tasks, as both were used by SLTs $50 \%$ or more of the time with a high and moderate level of consistency respectively. 'Real-life' tasks (not listed in the questionnaire) and 'barrier' tasks are also discussed as SLTs mentioned both during the focus group.

\section{Conversation Tasks}

In line with the questionnaire results, all focus group participants stated that conversation tasks formed a part of their CST. P2, P4 and P5 use conversation tasks in every session, and everyone (apart from P1) agreed that this task could last the entire session. Descriptions of what constituted a conversation task differed; for example, P2's description included spontaneous topic shifts: "You can start out in one area, and then that's going into monarchy and into history and then we may go into politics and you know, who knows where that's going to go you just got to let it go" (00:12:27 P2). However 13 of the 28 references did not appear to allow for "spontaneous shifts in topic or task, or elaboration beyond the task demands" (Simmons-Mackie, Savage, \& Worrall, 2014, p. 2). For example, P4 described a conversation with a client who "came from $X$, so I got a map of $X$ and he has to give me information that I did not know about from $X^{\prime \prime}$ (00:02:23 P4). Such comments were sub-categorized as 'structured communication tasks' (see Appendix C for definition). Despite these differences focus group members agreed with the moderator's summary that key to these tasks is "novel information" to ensure "it is a real conversation" (00:11:46 Moderator). 
As for education tasks, figure 9 shows that collaboration was the most mentioned technique used during conversation tasks (14/28 references), with six of the eight SLTs referring to the $\mathrm{CP}$ as the collaborator. However the frequency of CP involvement varies. P2 describes the partner as present "most of the time" (00:27:23 $\mathrm{P} 2)$ reflected in her description of having "a three way conversation" (00:07:54 P2), and P5 prioritises acute patients who have partners who visit. In contrast, P1 commented that he only "occasionally" brings in another person, as despite being "quite keen to get the partner involved... a lot of the time it just does not happen unfortunately" (00:27:27 P1).

Figure 9 here

Figure 9 shows the second most mentioned technique is 'feedback', mainly provided directly to the PwA about their communication strategy use. Although P1 discusses using video feedback, so that clients are "less relying on me to tell them" (00:14:07 P1), other examples of feedback during conversation tasks constitute verbal feedback from the SLT to a PwA, of which two forms emerge - delayed and immediate. Delayed feedback is reportedly used at the end of conversation tasks, focusing on "the strategies that have been used within that conversation, kind of emphasising the positive use of them" (00:16:22 $\mathrm{P} 8)$. The rationale for delayed feedback appearing to be that it then "doesn't feel like they've done work" (00:13:00 P5).

Immediate feedback within conversation tasks consists of SLTs either reinforcing a PwA's positive strategy use, "Oh Mr X, that's really good, you did that it worked you know (thumbs up gesture)" (00:27:47 P4), or requesting a PwA for feedback on a CP's strategy use "And how does it make you feel? You know, knowing the person is smiling and nodding even though they might have difficulty understanding you?" (00:04:10 P8). SLTs rationale for immediate feedback was to aid memory and online reflection about difficulties, "because otherwise you lose the moment" (00:16:07 P4).

The third most frequently mentioned technique used in conversation tasks is modeling (7/28 references). SLTs' references to modeling reveal a primary focus on the CP's behaviour. Six of the seven references provide examples of the SLT 
"exploring with the patient what's going to work" (00:47:24 P2) so that the CP can "kind of see it" (00:20: 25 P5).

The fourth technique, problem solving (6/28 references) is mainly used to stimulate clients' thinking about "which things" they would "like to specifically focus on" (01:00:46 P8) within therapy i.e. goal setting is the aim, and the technique used to get there is problem solving. For example posing the question "is there a way we could have done that easier" (00:15:12 P1).

The fifth technique, environmental restructuring (5 references) includes small environmental adjustments such as "putting a pen and paper there, not even saying it” (00:30:44 P6). Reflective techniques (4 references) include questions designed to elicit clients" on-line judgments about their performance "have you followed everything, that, you know, Mrs X has said to you?" (00:05:15 P8) and their emotional reactions to the consequences of their communication strategy use "how does that make you feel?" (00:04:10 P8). However reflection is also used by P2 to initiate a couple's practice conversation topic, asking them to reflect on shared experiences as a way of "bringing up stories" (00:07:09 P2).

Finally, the last two techniques, prompting and bridging (1 reference each), include P2 using forced choices to prompt a response from a PwA (00:09:26 P2) and P5 explicitly linking of "all the strategies we've been using" (00:13:00 P5) to the PwA's immediate conversational success with the SLT.

\section{$\underline{\text { Real Life Tasks }}$}

'Real-Life Tasks' was not an option in the questionnaire but emerged from the focus group data. Defined as 'a task where a client has to perform a functional activity within his or her natural environment' (Appendix C), it was first mentioned as an alternative to role-play tasks. P1 commented that he does "as much kind of real stuff as possible" (00:23:44 P1). P2, P3, P4, P5, and P8 then provided examples of similar tasks such as taking clients' to a coffee shop to practice ordering, and phoning a utility company during therapy. Figure 9 shows a breakdown of the 15 references to techniques used in these tasks. Environmental restructuring (definition in Appendix 
D) was the most frequently mentioned technique (6/15 references). Examples of this technique included SLTs creating a communicative need and ensuring environmental prompts were in place, including a communication strategy sheet acting "as a kind of visual reminder" (00:22:36 P1). Finally, examples included using the CP to prime the environment "it's the carer or partner who'll go in and clue in a shopkeeper and 'so he's going to come in with a £20 note and er, he doesn't talk but he understands just fine, you know, can you help him out?"' (00:29:33 P2).

The second most referenced technique, collaboration (4/15 references), included P5's mention of "joint sessions with the occupational therapist" (00:54:39 P5) and clients' asking for things "from the lady in the kitchen" (00:26:04 P5) as well as SLTs working with staff in "a little café under our stroke unit"(00:24:50 P3) and the CP who will "go in and clue in a shopkeeper"(00:29:33 P2).

The last two techniques prompts (2/15 references) and problem solving (1/15 references) describe specific instructions within a task "I want you to come up with what it is. I want you to tell the CP what to get for me" (00:54:39 P5) and "You have to ask" (00:24:25 P2) and P5's talking about how a PwA will continue her visits to the hairdressers asking "Who books the appointment for you?" followed by "How do you think you are going to be able to do that now?" (00:20:26 P5).

In summary, real-life tasks were a new task type described by SLTs within the focus group. The two most used techniques within these tasks are environmental restructuring and collaboration.

\section{Picture Description and Barrier Tasks}

Finally the focus group explored the use of picture description tasks in CST. This revealed a blurring of boundaries for some of the group between picture description and barrier tasks. As a result, these two tasks will be discussed together.

Although participants agree that they use picture description tasks, it appears that picture description is often used at the start of intervention "as an outcome measure" 
(00:28:49 P7) capturing "the amount of information" a PwA is able to convey, "with their strategies" (00:28:49 P7).

However the blurring of boundaries between picture description and barrier tasks is visible when P5 further comments “yeah it's like a demonstration with a barrier game but kind of combined really" (00:31:03 P5). P7 also comments "I've done a similar thing, where I would do a sort of barrier task with a picture description with the patient" (00:47: $41 \mathrm{P} 7)$. P2 provides a definition of a barrier task as "based on erm constraint induced" (00:31: 55 P2), which appears to be a reference to Intensive Language Action Therapy (ILAT) principles (see Pulvermüller \& Berthier, 2008).

The focus group also revealed CP attendance at sessions to be lower than SLTs would like, illustrated well by one participant's comment "a lot of the time it just does not happen unfortunately" (00:15:12 PH). It also uncovered a trend towards implicit training techniques with a $\mathrm{CP}$ and explicit training techniques with a PwA. Implicit techniques aim to indirectly modify target behaviours (Simmons-Mackie, Savage, \& Worrall, 2014) and are reflected in SLTs' frequent descriptions of modeling within education, and conversation tasks to educate CPs about optimal communication strategies. In contrast, explicit techniques aim to overtly modify behaviour using direct discussion of target behaviours and goals (Simmons-Mackie, Savage, \& Worrall, 2014). This study suggests that explicit techniques are more often directed at the PwA and implicit techniques at the CP, one SLT commenting, "many partners are not into doing the actual tasks" (00:44:11 P2) and another "I have realized as I am completing this survey that I don't do this [discuss and write down communication strategies] as much with the CP and I am wondering why this is?!" (SLT 739356 Q.35). SLTs in the focus group described the provision of targeted verbal feedback regarding the successful use of communication strategies in conversation tasks for PwA more than for CPs. They also described actively prompting a PwA to use specific communication strategies within barrier tasks. This is not to say implicit and explicit techniques are mutually exclusive to the $\mathrm{CP}$ and PwA respectively.

(iii) The use of video in CST 
Finally the focus group explored the reasons behind SLTs' highly consistent non-use of video. As figure 10 shows extrinsic and intrinsic barriers to video use were identified, with extrinsic barriers the most frequent reasons for non-use. Barriers range from difficulties retaining video equipment on wards, to internal concerns about personal technology skills.

Figure 10 here

\section{Conclusions \& Implications}

This study reports on current UK SLT practices when undertaking CST in aphasia rehabilitation, in the absence of such studies in the UK and the availability of published CST approaches for SLTs. Three key CST findings arise from this study: (i) the rarity with which SLTs focus equally and explicitly on both communication partners' strategies (ii) SLTs' differing understandings of CST terminologies and concepts and underuse of formal assessment (iii) the absence of video feedback. The clinical and research implications of these findings will be discussed below.

This study's survey findings suggest that CPs not only receive half the amount of CST given to PwAs, but they also play a more passive learning role when they are present. This is an interesting consideration when the current evidence base contains stronger evidence for the effectiveness of CP-CST over other CST approaches, describing it as "an effective method" that "may be maintained over time" (Simmons-Mackie et al., 2010, p. 1833). However this inequality is not surprising as CPs absence from CST was a key finding of the Johansson, et al (2011) and Hallé, et al (2014) studies. Comments from our focus group suggested SLTs felt CPs were often reluctant to participate. However one questionnaire participant expressed surprise on realizing this inequality existed in their own practice, suggesting CP reluctance may not be the complete story. While the rationale for a CP's presence/absence in CST was not the focus of this study, Hallé et al (2014) revealed SLT concerns that CST may overburden CPs, damage their sense of competence as carers and prompt awkward conversations when CPs may be focused on a PwA's full recovery. It is possible that these concerns might also explain the strategic decision that SLTs in this study appear to have made to use more implicit techniques 
with a CP. Implicit techniques, such as modeling, avoid awkward discussions about adaptation versus recovery, and about $\mathrm{CP}$ strategies that hinder the PwA, whilst simultaneously reducing the burden of direct practice. However, the inequality in approach may also reflect the fact that SLTs feel a lack of license to modify the communicative behaviours of someone without a communication disorder. These are important issues, requiring further study in the UK.

The second key finding, namely SLTs' differing understandings of key CST concepts and terminology and the underuse of formal assessment, suggests differences between research and clinical practice. It is possible that the poor uptake of formal assessment may relate to a fear of the time investment and skill required for qualitative analysis, but this issue was not explored during the study so remains speculative. Interestingly, study participants' descriptions of conversation tasks did not always encapsulate the unstructured and spontaneous nature of conversation tasks as defined in the literature (Beckley et al., 2013; Simmons-Mackie et al., 2014; Wilkinson, 2010). Rather, the descriptions sub-categorised 'structured conversation task' had many characteristics of an institutional interaction, with SLTs leading the task using props and questions (Wilkinson, 2010) and reinforcing their status as the 'expert' or authority within the task through their use of evaluative feedback (Simmons-Mackie \& Damico, 2007). Such actions may affect a PwA's conversational behaviours, since we know that institutional interactions are one-sided in terms of topic and questioning (Wilkinson, 2010). It could be argued that this denies them full participation within the interaction (Simmons-Mackie \& Damico, 2007), a position that directly contradicts the desire of participants in this study to have 'real' conversations where a PwA feels in control and able to express an opinion. This mismatch highlights the importance of researchers continuing to engage clinicians in a dialogue about their clinical practice, to ensure incorporation of key research findings into clinical practice.

The absence of video feedback from clinical practice was the third key finding. Research suggests that video provides an opportunity to empower a PwA and CP to develop their own insight and opinion into their communication strategy use (Beckley et al., 2013). However, only one focus group participant (P1) made an 
explicit link between the use of video and empowering clients to evaluate of their own performance. Other participants agreed that video feedback was desirable but cited multiple barriers to its use including equipment theft, legal issues and client readiness. These findings suggest that clinicians agree with researchers about the powerful nature of video as a therapeutic tool, but its implementation is currently extremely difficult. This dichotomy raises an important question about whether the area of video feedback, should continue to be advocated in this context.

The limitations of this study need to be acknowledged. SLTs were recruited to the study using purposeful sampling, which may be considered a limitation that future research should address. In addition results are based on 37 questionnaire responses and a single focus group, which was not submitted for membership checking thus they may not fully represent UK SLTs' practices in this area.

In summary, this study suggests that, despite the evidence base for the effectiveness of CP training (Simmons-Mackie et al., 2010) and the growing evidence base for an equal focus on training both a PwA and CP (Wilkinson \& Wielaert, 2012), the reality of clinical practice means CST is focused more on the strategy use of a PwA. These findings reinforce the importance of investigating how practicing SLTs carry out CST (and indeed all therapies) and how researchers can best meet clinical need. Future studies may include correlating techniques used frequently by SLTs with effectiveness and greater consideration of the context of therapy delivery in the design of future CST interventions (Johnson, 2015; Michie, van Stralen, \& West, 2011).

\section{Acknowledgments}

A full version of the questionnaire is available from the first author. Thanks go to Dr Steven Bloch, Ms Nicola Sirman and Dr Merle Mahon (University College London) for their advice regarding questionnaire development.

\section{References}

Armstrong, Ferguson, \& Simmons-Mackie. (2013). Discourse and Functional Approaches to Aphasia in (2013). In L. Papathanasiou, P. Coppens, \& C. 
Potagas (Eds.), Aphasia and Related Neurogenic Communication Disorders. Jones and Bartlett Learning.

Bandura, A. (1994). Self-Efficacy. In V. . Ramachaudran (Ed.), Encyclopedia of human behavior (pp. 71-81). New York: Academic Press.

Beckley, F., Best, W., Johnson, F., Edwards, S., Maxim, J., \& Beeke, S. (2013). Conversation therapy for agrammatism: exploring the therapeutic process of engagement and learning by a person with aphasia. International Journal of Language \& Communication Disorders / Royal College of Speech \& Language Therapists, 48(2), 220-39. doi:10.1111/j.1460-6984.2012.00204.x

Beeke, Beckley, F., Johnson, F., Heilemann, C., Edwards, S., Maxim, J., \& Best, W. (2015). Conversation focused aphasia therapy: Investigating the adoption of strategies by people with agrammatism. Aphasiology, 29(3), 355-377. doi:10.1080/02687038.2014.881459

Beeke, S., Sirman, N., Beckley, F., Maxim, J., Edwards, S., Swinburn, K., \& Best, W. (2013). Better Conversations with Aphasia: an e-learning resource. UCLeXtend.

Brown, K., Worrall, L. E., Davidson, B., \& Howe, T. (2012). Living successfully with aphasia: a qualitative meta-analysis of the perspectives of individuals with aphasia, family members, and speech-language pathologists. International Journal of Speech-Language Pathology, 14(2), 141-55. doi:10.3109/17549507.2011.632026

Davis, G. ., \& Wilcox, M. . (1985). Adult aphasia rehabilitation: Applieed pragmatics. College-Hill Press.

Fereday, J., \& Muir-Cochrane, E. (2006). Demonstrating Rigor Using Thematic Analysis : A Hybrid Approach of Inductive and Deductive Coding and Theme Development. International Journal of Qualitative Methods, 5(1), 80-92.

Hallé, M.-C., Le Dorze, G., \& Mingant, A. (2014). Speech-language therapists' process of including significant others in aphasia rehabilitation. International Journal of Language \& Communication Disorders / Royal College of Speech \& Language Therapists, 49(6), 748-60. doi:10.1111/1460-6984.12108 
Johansson, M. B., Carlsson, M., \& Sonnander, K. (2011). Working with families of persons with aphasia: a survey of Swedish speech and language pathologists. Disability and Rehabilitation, 33(1), 51-62. doi:10.3109/09638288.2010.486465

Johnson, F. (2015). What works in conversation therapy for aphasia, and how? Searching for mechanisms of change and active ingredients using tools and theory from behaviour change research. Unpublished PhD thesis. University College London.

Kagan, A. (1998). Supported conversation for adults with aphasia: methods and resources for training conversation partners. Aphasiology, 12(9), 816-830. doi:10.1080/02687039808249575

Kagan, A. (2014). Rating Support and Participation in Conversation (MSC \& MPC). Aphasia Institute Website.

Lock, S., Wilkinson, R., \& Bryan, K. (2001). Supporting partners of people with aphasia in relationships and conversation (SPPARC). International Journal of Language \& Communication Disorders, 36(Supplement), 25-30. doi/abs/10.3109/13682820109177853

Mathers-Schmidt, B. a, \& Kurlinski, M. (2003). Dysphagia evaluation practices: inconsistencies in clinical assessment and instrumental examination decisionmaking. Dysphagia, 18(2), 114-25. doi:10.1007/s00455-002-0094-z

Michie, S., van Stralen, M. M., \& West, R. (2011). The behaviour change wheel: a new method for characterising and designing behaviour change interventions. Implementation Science : IS, 6(1), 42. doi:10.1186/1748-5908-6-42

Murphy, M., Black, N., Lamping, D., McKee, C., Sanderson, C., Askham, J., \& Marteau, T. (1998). Consensus development methods, and their use in clinical guideline development. Health Technology 2(3).

Pulvermüller, F., \& Berthier, M. L. (2008). Aphasia therapy on a neuroscience basis. Aphasiology, 22(6), 563-599. doi:10.1080/02687030701612213

Purdy, Duffy, R., \& Coelho, C. (1994). An investigation of the communicative use of trained symbols following multimodality training. Clinical Aphasiology, 22, 
$345-356$.

Rautakoski, P. (2011). Training total communication. Aphasiology, 25(3), 344-365. doi:10.1080/02687038.2010.530671

Royal College of Physicians Intercollegiate Stroke Working Party. (2012). National clinical guideline for stroke 4th edition.

Simmons-Mackie, N. (2008). Social Approaches to Aphasia Intervention. In R. Chapey (Ed.), Language Intervention Strategies in Adult Aphasia (5th ed., pp. 290-317). Lippcott Williams \& Wilkins.

Simmons-Mackie, N., \& Damico, J. S. (1997). Reformulating the definition of compensatory strategies in aphasia. Aphasiology, 11(8), 761-781.

Simmons-Mackie, Raymer, A., Armstrong, E., Holland, A., \& Cherney, L. R. (2010). Communication partner training in aphasia: a systematic review. Archives of Physical Medicine and Rehabilitation, 91(12), 1814-37. doi:10.1016/j.apmr.2010.08.026

Simmons-Mackie, Savage, M. C., \& Worrall, L. (2014). Conversation therapy for aphasia: a qualitative review of the literature. International Journal of Language \& Communication Disorders, 49(5), 511-526. doi:10.1111/14606984.12097

Simmons-Mackie, N. N., \& Damico, J. S. (2007). Access and social inclusion in aphasia: Interactional principles and applications. Aphasiology, 21(1), 81-97. doi:10.1080/02687030600798311

Wilkinson, R. (2010). Interaction-focused intervention: A conversation analytic approach to aphasia therapy. Journal of Interactional Research in Communication Disorders, 1(1), 45-68. doi:10.1558/jircd.v1i1.45

Wilkinson, R., Lock, S., Bryan, K., \& Sage, K. (2011). Interaction-focused intervention for acquired language disorders: facilitating mutual adaptation in couples where one partner has aphasia. International Journal of SpeechLanguage Pathology, 13(1), 74-87. doi:10.3109/17549507.2011.551140

Wilkinson, R., \& Wielaert, S. (2012). Rehabilitation targeted at everyday communication: can we change the talk of people with aphasia and their 
significant others within conversation? Archives of Physical Medicine and Rehabilitation, 93(1 Suppl), S70-6. doi:10.1016/j.apmr.2011.07.206

Yoshihata, H., Watamori, T., Chujo, T., \& Masuyama, K. (1998). Acquisition and generalization of mode interchange skills in people with severe aphasia. Aphasiology, 12(12), 1035-1045. doi:10.1080/02687039808249468

Appendix A-D here 